\title{
Recommender System Challenges and Methodologies in Social Network: Survey
}

\author{
Himgauri D. Ambulkar ${ }^{1}$, Apashabi Pathan ${ }^{2}$ \\ ${ }^{1}$ Savitribai Phule Pune University, G. H. Raisoni College of Engineering, Dhomkhel Road, Wagholi, Pune 412207, Maharashtra \\ ${ }^{2}$ Professor, Savitribai Phule Pune University, G. H. Raisoni College of Engineering, Dhomkhel Road, Wagholi, Pune 412207, Maharashtra
}

\begin{abstract}
Recommender system aims to provide user with guidance related to the useful services based on their personalized service recommendation, Past behaviors or neighbor's preferences. With the popularity of social network, many users like to share their views on social media such as rating, blogs, tweets etc, which helps to recommend users interest items. Service recommender system provides an appropriate recommendation of services like shopping, gadgets, hotels, movies etc, which produces big data on the web. This results in the problem of information overload. To get over this problem, Personalized Recommendation System have been affluently used. This paper presents a survey on various challenges and methodologies in recommendation system.
\end{abstract}

Keywords: Recommender System, Social Network, Collaborative Filtering, Interpersonal Interest

\section{Introduction}

Recommender systems is the standard device which attempt to recommend the most suitable items ( services) to specific users (individuals or businesses) by judging a user's interest in an item found on related information about the items, the users and the communication between items and users [1]. Recommendation system (RS) has been successfully utilized to solve difficulty of information overload. The aim of developing recommender systems is to condensed information overload by retrieving the most suited information and services from a large amount of data, thereby providing personalized services. Survey shows that more than 20 percent of sales generated through recommendation. Most of the users believe that items recommended by friend or neighbors are useful [2] and very few buy the recommended products or items of their personal interest.

The most important feature of a recommender system is its ability to "predict" a user's interests by observing the behavior of this user and the behavior of other users to generate personalized recommendations [3]. In a large web space, recommendation support to find items of user interest [6]. Recommendation system commonly used two methods based on how user profile information is used:

1)Content-based : The recommender will retrieve items whose content is match to those of the profile.

2)Collaborative Filtering: is based on opinions of other users

- Memory based (User-based): compare text documents to user profiles

- Model based (Item-based): use data mining techniques to develop a model of user ratings. [5]

The cold start problem is related to recommendations for novel users or new items. In case of new users, the system does not have information about their preferences in order to make recommendations [7]. Cold start has been a serious problem for Data Mining works. Cold start can be described as unavailability of data for modeling algorithms [6]. Web is not static, so it very difficult to predict the user interested items [4] in time.

Personalized RS combines social factors such as interpersonal interest similarity, personal interest and interpersonal influence [2]. Personalized RS is useful to recommend the items on social networks with the aim that recommended items should based on their past behavior and interpersonal relationship of social networks. [8] Propose a good approach to improve the accuracy of recommendation system by introducing the concept of "inferred circles of friends". Interpersonal interest of user in social network helps to improve the quality of the recommendation [9]. Accuracy of recommendation can be improved with the help of several social-trust based RS. The problem of cold start and sparsity can be solved with the help of interpersonal relationship in the circle of friends, of social networks and social contexts [10]

\section{Recommender System Challenges}

\section{A. Cold Start problem}

Cold start problem refers to the situation where an item cannot be recommended unless it has been rated by a substantial number of users. [6].The cold start problem has been occurring due to rapidly increasing of registered users and various products [11]. From [11] cold start problems have three types: new user problem, new item problem and new system problem. In case of new user, the information about the user is not sufficient. Hence it is very hard to provide recommendation. Collaborative filtering is unable to perform useful recommendations for a new item and new user, because ratings are usually unavailable. Generally user rate the small amount of products, hence sparsity problem is observed with a large set of items. It is difficult to find the pattern [15] in case of new system, due to availability of very less information about user and newly entered product or items. Interpersonal relations such as "friend circle"[2] and individual preferences are very important factor in social network , which helps to solve the problem of cold start. [17] Propose to recommend apps to the Twitter users by applying latent Dirichlet allocation to produce latent groups based on 


\section{International Journal of Science and Research (IJSR) \\ ISSN (Online): 2319-7064}

Index Copernicus Value (2013): 6.14 | Impact Factor (2014): 5.611

the users' followers, in order to solve the problem of cold start app recommendation. Work [19] tries to show the coldstart problem as a ranking task by introducing a pair wise preference regression model and thus minimizing the distance between the real rank of the items and the approximated one for each user.

\section{B. Scalability}

Scalability problem in recommendation is caused due to the increasing number of users, group users into clusters based on their preferences toward similar data objects such as movies, jokes, etc [12]. Scalability is the property of system which handle emergent amount of information [15] in a good manner. With rapid growth in use of internet, the recommender systems are having an explosion of data [10] and thus it is a serious problem to handle with constantly growing demand. The computation in recommender system algorithms helps to increase the growing number of items and users. The Method which solve scalability problems are based on approximation mechanism [22]. Collaborative filtering, clustering and pre-processing are used to solve Scalability problem [15][21]. Noisy data is cleaned and transformed [13] to the recommendation system in preprocessing. With the help of Clustering similar items can be grouped[21] with intra cluster distance as small as possible and inter cluster distance as large as possible. Scalability is serious problem since CF systems often have to manage large number of users or items. Used based or memory based collaborative filtering uses the user's profile. Personalized recommendation algorithm [2] also used to solve the problem of scalability.

\section{Sparsity Problem}

Sparsity problem is one of the major problems encountered by recommender system is data sparsity has great influence on the quality [21] of recommendation. The main reason behind data sparsity is that most users do not rate most of the items and the available ratings are usually sparse. In collaborative filtering [22] technique it is important that the more users are required to be rated the item. Though high rating [6] given by few users' leads to problem of sparsity. To prevail over the sparsity problem, one can use user profile information [6] while calculating user similarity item with others. Similarity in users can be identified with the aid of age, area code, gender, demographic segment etc. Sparsity problem also resolved by associative retrieval framework and related spreading activation algorithms [10]. Sparse rating matrix can used to resolve the sparsity problem. Item based mining and associative retrieval technique [5] also used to overcome the problem of sparsity.

\section{Privacy}

Many user do not want to share data about themselves due to issues of privacy. Vulnerable architecture is the problem of CF system, Due to which privacy of individuals can not be preserved. It is very tough task to gain proper and truthful amount of information for CF purposes, Because of privacy concerns. User feel more trustable and happy to share their data, If user is assured that privacy will be maintained. With rapid use of internet, big amount of data is shared on internet, thus Privacy is becoming challenge of recommendation system.

\section{E. Overspecialization}

It prevents user from discovering new items and other available options [15]. However, diversity of recommendations is a desirable characteristics [6] [11] of all recommendation system. User will be given with a set of different and a wide range [15] of alternatives, After solving the problem using genetic algorithms. If system recommends only those items which are having high rating against the user profile, In that case Overspecialization occurs. For example if a user has no experience with Indian foods will never get recommendation though the Indian restaurant are available in the city. The problem of overspecialization can be solved using similarity fusion and probabilistic fusion [13]. Sampling, similarity measures, and dimension reduction in collaborative filtering [14] helps to overcome the problem of overspecialization.

\section{Personalized Recommendation System Approach In Social Network}

Content based filtering and item based filtering are very popular methods of Recommendation. Both of these systems are accessible to the problem of sparsity and cold start. Recommendation system uses interpersonal interest social profile to recommend user interested items to solve these problems. Most of the user likes to buy products recommended by their friends, thus personalized recommendation systems helps to make decision. Some of the approaches of personalized recommendation are discussed below.

\section{A. Basic Matrix Factorization}

The Basic Matrix factorization is combined with social network data in a recommendation system [2] where, the probabilistic matrix factorization does not take any social factor into consideration. The task of RS is to decrease the error of predicted value to the real rating value. Thus, the Base MF model is trained [2] [21] on the observed rating data by minimizing the objective function. The objective function can be minimized using gradient descent method. The matrix factorization decomposes a $\mathrm{M}$ matrix into the product of several factor matrices i.e. $\mathrm{M}=\mathrm{F} 1 \mathrm{~F} 2$...Fn [21] where $n$ can be any number but usually it is 2 or 3.Due to superior performance in scalability, Base matrix factorization has gained more popularity over several years [11]. Whenever connection between variable and observed variable is estimated during the training recommendation can made by computing possible interaction [16] with each product in separate matrix, called base matrices.

\section{B. Circle Con Model}

The Circle Con model [8] has been found to outperform BaseMF and SocialMF with respect to accuracy of the RS. The approach focuses on the factor of interpersonal trust in social network and infers the trust circle. The matrix represents trust value of user-user. Furthermore, the whole trust relationship in social network is divided into several sub-networks, called inferred circle and each circle is related to a single category of items. Circle Con model can be processed in the following steps [8]. First, trust circle inference, this can be recognized with the aid of different 


\section{International Journal of Science and Research (IJSR) \\ ISSN (Online): 2319-7064}

Index Copernicus Value (2013): 6.14 | Impact Factor (2014): 5.611

categories with certain threshold value. Second, trust value assignment, this can be done with equal trust, enterprise based trust and trust splitting. Social contexts integrated [18] to give more accurate results. Rating and review is very important in a large scale social network, To recommend the movies to other users based on the user profile, flixster [22] uses this model. They allow the users to rate and review the movies based on their interest, and collects the information. Over large sets of data CircleCon model gives the high quality[23] of recommendation in social network.

\section{Context MF Model}

Jiang et al. [18] demonstrate the significance of social contextual factors (including individual preference and interpersonal influence) for item adopting on real Twitter and Facebook style datasets. The task of ContextMF model in [18] is to recommend acceptable items from sender to receiver. Here, the factor of interpersonal influence is similar to the trust values in CircleCon model [8]. Moreover, individual preference is mined from receiver's historical adopted items. User rated items has more influence than individual preference in ContextMF model, because it easier for the recommended items of our model to be transformed into purchase rate than the adopted items[23] in Facebook style social networks. It is observed that neighbors in the social network have similar interest,Context matrix factorization helps to identify similar interest by training objective function [8]. Direct neighbors can be identified by Bayesian inference [22] which helps to identify user personal interest directly related [2] to rated items. Individual interest and inter personal interest are considered in context matrix factorization because it is easy to recommend the user interested items in real time. The performance of the cold start is improved [22] by 50\% using ContextMF.

\section{Conclusion}

This survey paper explains the different challenges and methodologies used to solve the recommendation system challenges in social network with user interest. This survey paper also described different methodologies used to build recommendation system along with the user's personal interest and interpersonal interest in social network by social matrix factorization and base matrix.

\section{References}

[1] J. Bobadilla, F. Ortega, A. Hernando, A. Gutiérrez, Recommender systems survey, Knowledge-Based Systems, 46 (2013) 109-132

[2] X Qian, H Feng, G Zhao, $T$ Mei "Personalized Recommendation Combining User Interest and Social Circle", Knowledge and Data Engineering, IEEE Transactions, 2014

[3] P. Resnick, H.R. Varian, Recommender systems, Communications of the ACM, 40 (1997) 56-58

[4] H.R. Kim and P.K. Chan. "Learning Implicit User Interest Hierarchy for Context in Personalization". In Proc. of International Conference on Intelligent User Interface, 2003.
[5] Mozhgan Tavakolifard, Kevin C. Almeroth, "Social Computing: An Intersection of Recommender Systems, Trust/Reputation Systems, and Social Networks", IEEE Network, July/August 2012, Vol. 26, No. 4, pp. 53-58

[6] M. Jahrer, A. Toscher, and R. Legenstein. "Combining predictions foraccurate recommender systems". KDD'10, pp. 693-702, 2010.

[7] Blerina Lika,Kostas Kolomvatsos,Stathes Hadjiefthymiades,"Facing the cold start problem in recommender systems", An International Journal archive Volume 41 Issue 4, March, 2014

[8] X. -W. Yang, H. Steck, and Y. Liu. "Circle-based recommendation in online social networks”. KDD'12, pp. 1267-1275, Aug.2012

[9] R. Sinha, and K. Swearingen, "Comparing recommendations made by online systems and friends". Dublin, Ireland, 2001

[10] Xavier Amatriain "Mining Large Streams of User Data for Personalized Recommendations" ACM SIGKDD Explorations, 2012.

[11] H. Ma, I. King, and M. R. Lyu. "Learning to recommend with social trust ensemble". SIGIR'09, 2009

[12] Braak, P.t., Abdullah, N., Xu, Y.: Improving the Performance of Collaborative Filtering Recommender Systems through User Pro?le Clustering. In: The 2009IEEE/ACM Int'l Joint Conference on Web Intelligence and Intelligent Agent Tech-nology, vol.03, pp. 147-150. IEEE Computer Society, Washington (2009)

[13] J. Wang, A.P. de Vries, and M.J.T. Reinders. "Unifying user-based and item-based collaborative filtering approaches by similarity fusion”. SIGIR'06, 2006.

[14] O.Phelan, K. McCarthy, and B. Smyth. "Using twitter to recommend realtime topical news." In Proceedings of the third ACM conference on Recommender systems, pp. 385-388. ACM, 2009

[15] G. Adomavicius, and A. Tuzhilin. "Toward the next generation of recommender systems: a survey of the state-of-the-art and possible extensions". Knowledge and Data Engineering, IEEE Transactions on, pp. 734749, Jun. 2005.

[16] H Ma, D Zhou, C Liu, MR Lyu, I King. "Recommender Systems with Social Regularization". Fourth ACM international conference on Web search and data mining, 2011.

[17] J. Lin, K. Sugiyama, M.-Y. Kan, and T.-S. Chua. Addressing cold-start in app recommendation: Latent user models constructed from twitter followers. In SIGIR'13, pages 283-293, 2013

[18] M. Jiang, P. Cui, R. Liu, Q. Yang, F. Wang, W. -W. Zhu and S. -Q. Yang. "Social contextual recommendation". CIKM'12, pp. 45-54,2012

[19] S.-T. Park and W. Chu. Pairwise preference regression for cold-start recommendation. In RecSys'09, pages 21-28,2009

[20] L Sharma, A Gera "A Survey of Recommendation System: Research Challenges" International Journal of Engineering Trends andTechnology, 2013

[21] R. Salakhutdinov and A. Mnih. "Probabilistic matrix factorization". In NIPS 2008, 2008

\section{Volume 4 Issue 11, November 2015}




\section{International Journal of Science and Research (IJSR) \\ ISSN (Online): 2319-7064}

Index Copernicus Value (2013): 6.14 | Impact Factor (2014): 5.611

[22] M. Jamali and M. Ester. "A matrix factorization technique with trust propagation for recommendation in social networks". In Proc.ACM conference on Recommender systems (RecSys), 2010.

[23] H. Ma, H. Yang, M.R. Lyu, and I. King. "Sorec: Social recommendation using probabilistic matrix factorization”.CIKM'08, 2008. 